\begin{tabular}{c|c|c|} 
CRITICAL & $\begin{array}{l}\text { Western Ghats } \\
\text { EARTNERSHIP FUND }\end{array}$ \\
Special Series
\end{tabular}

\section{Birds of the SÁlim Ali Centre for ORNITHOLOGY AND NATURAL HISTORY CAMPUS, ANAIKATTY HILLS, SOUTHERN INDIA}

\section{A. Mohamed Samsoor Ali ${ }^{1}$, S.B. Shanthakumar ${ }^{2}$, S. Ramesh Kumar ${ }^{3}$, R. Chandran ${ }^{4}$, S. Suresh Marimuthu ${ }^{5} \&$ P.R. Arun $^{6}$}

1,2,3,4,6 Environmental Impact Assessment Division

${ }^{5}$ Ornithology Division, Sálim Ali Centre for Ornithology and Natural History (SACON), Anaikatty, Coimbatore, Tamil Nadu 641108, India

${ }^{1}$ amsamsoor@yahoo.co.in (corresponding author), ${ }^{2}$ srshanth@gmail.com,

${ }^{3}$ ramesh.wild@gmail.com, ${ }^{4}$ chandran.r.123@gmail.com,

${ }^{5}$ ssureshmarimuthu@yahoo.co.in, ${ }^{6}$ eiasacon@gmail.com

Birds are common dwellers of ecosystems and have been considered as environmental bio-indicators of inhabited areas (Blair 1999). There are approximately 9,990 bird species recorded in our planet and, the Indian subcontinent is home to 1,313 bird species (Grimmett et al. 2011). Tamil Nadu is known for its rich diversity of avifauna with more than 450 species including several endemic and conservation prioritised species (Islam \& Rahmani 2004). Previously, Nirmala (2002) documented the birds of the Anaikatty area of the Western Ghats. The present study attempts to prepare a checklist of birds in and around the campus of the Sálim Ali Centre for Ornithology and Natural History (SACON) situated in the Anaikatty Hills $\left(11^{\circ} 05^{\prime} 30.9^{\prime \prime} \mathrm{N} \& 76^{\circ} 47^{\prime} 36.2^{\prime \prime} \mathrm{E}\right), 30 \mathrm{~km}$ northwest of Coimbatore City, on the border of Nilgiri Biosphere Reserve (NBR), Western Ghats, Tamil Nadu
(Fig. 1). Being located on the eastern slope ( 600 elevation), the climate is semi-arid with temperature soaring up to $41^{\circ} \mathrm{C}$ during summer and the annual rainfall hardly exceeds $700 \mathrm{~mm}$. The SACON campus was established during early 1994 in 55 acres of degraded land. Tree planting initiatives

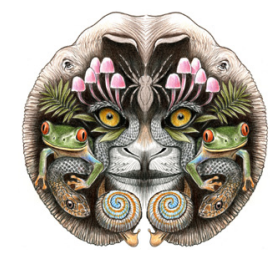

ISSN

Online 0974-7907 Print 0974-7893

\section{OPEN ACCESS} (in 2000) and protection measures against livestock grazing enhanced the vegetation growth inside the campus which became a source of attraction for many bird species. The campus' vegetation is predominantly scrub forest type, with a small Eucalyptus plantation. The campus harbours 200 species of plants that includes 60 tree species (Balasubramanian et al. 2012). Common woody species of the campus include Premna tomentosa, Albizia amara and Chloroxylon swietenia and tall shrubs include Lantana camara and Chromolaena odorata. Based on rainfall and temperature the study area experiences four distinct seasons, namely, southwest monsoon (June-August), northeast monsoon (September-November), winter (December-February) and summer (March-May).

Regular fortnightly surveys between January 2012 and May 2013 were done by walk on fixed routes in and around the campus including the banks of a nonperennial stream located on the western side of the campus. Surveys were usually made in the morning (07:00-10:00 hr) and evening (16:00-18:00 hr), and also sometimes in the night for owls and nightjars. The identification of birds in the field was based on Ali (2002) and Grimmett et al. (2011), while Manakadan \& Pittie (2001) was referred to for binomial names and

DOI: http://dx.doi.org/10.11609/JoTT.3660.5288-98 | ZooBank: urn:Isid:zoobank.org:pub:D4BAF57A-7EB2-42CB-959A-E081077B8BCD

Editor: C. Srinivasulu, Osmania University, Hyderabad, India.

Date of publication: 26 December 2013 (online \& print)

Manuscript details: Ms \# 03660 | Received 11 June 2013 | Final received 04 December 2013 | Finally accepted 08 December 2013

Citation: Ali, A.M.S., S.B. Shanthakumar, S.R. Kumar, R. Chandran, S.S. Marimuthu \& P.R. Arun (2013). Birds of the Sálim Ali Centre for Ornithology and Natural History Campus, Anaikatty Hills, southern India. Journal of Threatened Taxa 5(17): 5288-5298; http://dx.doi.org/10.11609/JoTT.3660.5288-98

Copyright: (c) Ali et al. 2013. Creative Commons Attribution 3.0 Unported License. JoTT allows unrestricted use of this article in any medium, reproduction and distribution by providing adequate credit to the authors and the source of publication.

Funding: None.

Competing Interest: The authors declare no competing interests.

Acknowledgements: Authors are thankful to Dr. P.A. Azeez, Director, SACON, for his immense support and allowing us to conduct this study. We grateful to Drs. Rajah Jayapal and P. Balasubramanian, Principal Scientists, SACON, for their valid comments and suggestions to improve the manuscript. 


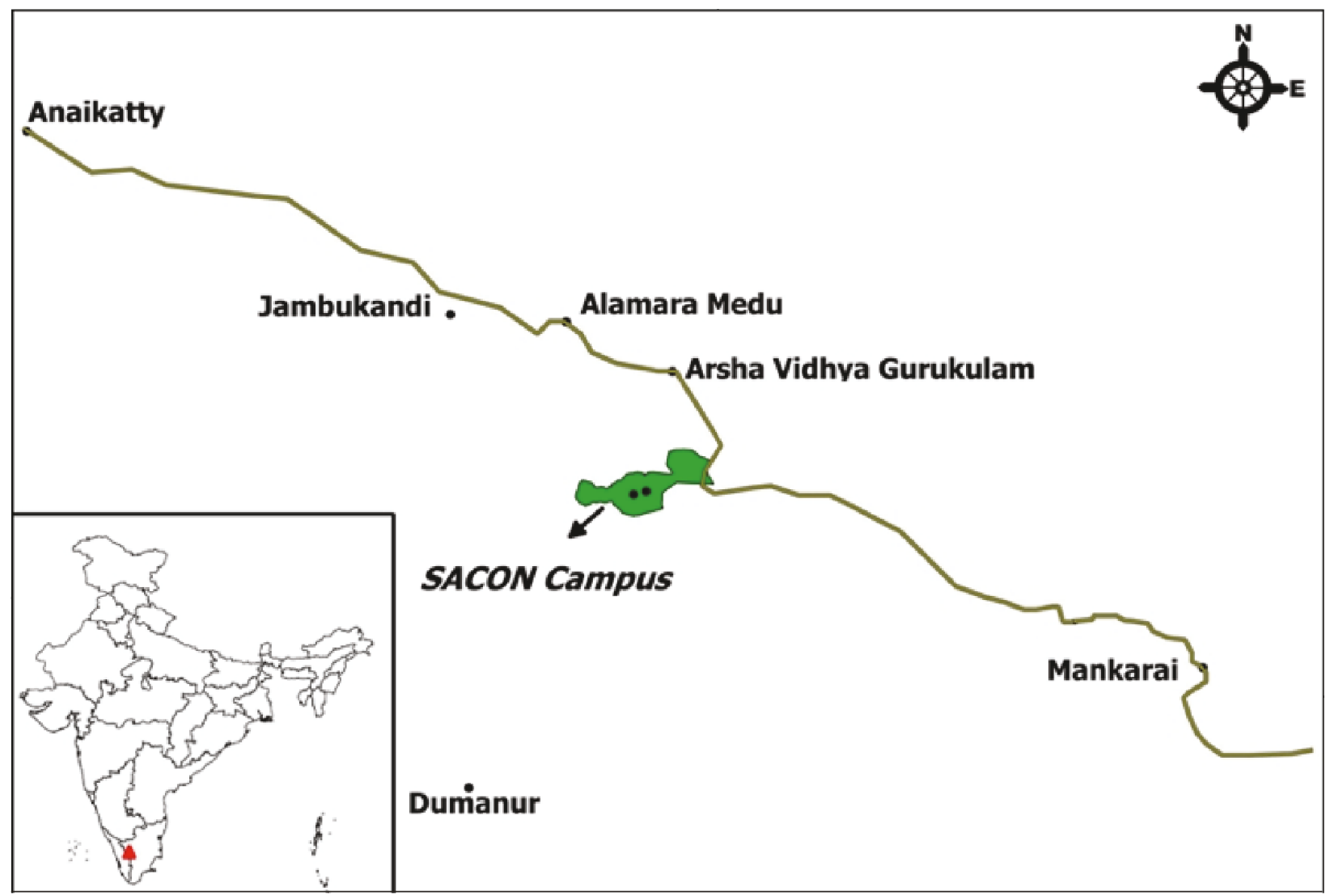

Figure 1. Location map of the Sálim Ali Centre for Ornithology and Natural History (SACON), southern India

authorities. The photographs of birds were taken with a SONY Digital Camera (DSC-HX200V) with zoom lens.

Depending on movement and seasonality of occurrence, the birds are classified as: resident (species found throughout the year), winter migrant (species migrates from outside India during winter) and local migrant (species found irregularly but is resident to India). Depending on the frequency of sightings during the field visits, birds are classified as: abundant (birds sighted in more than $80 \%$ of the visits), common (sighted in 60-<80\%), uncommon (sighted in 40-<60 $\%$ ), occasional (sighted in $20-<40 \%$ ) and rare (sighted in $<20 \%$ ). Breeding bird referred to as species observed to breed or showing evidence (nests or newly fledged chick(s) recorded) in the study area.

Results and Discussion: A total of 145 species of birds belonging to 106 genera and 48 families were recorded in and around the SACON campus (Appendix 1). The study area supports 76 passerine and 69 non-passerine bird species. Among the passerines, the best represented family is the Sylviinae (nine species), Timaliinae (seven species), Turdinae (six species) and Fringillidae and Dicruridae (five species each). Of the non-passerines, the Accipitridae (12 species) has the most species, followed by Cuculidae (eight species), Columbidae and Picidae (six species each) and Psittacidae and Alcedinidae (four species each). Two species, Blue-winged Parakeet Psittacula columboides and White-cheeked Barbet Megalaima viridis are endemic to the Western Ghats. Of the 145 species, all the 12 species belonging to the family Accipitridae, Indian Peafowl Pavo cristatus and Indian Grey Hornbill Ocyceros birostris are included under Schedule-I of the Indian Wildlife (Protection) Act, 1972.

Among the 145 species, 73 (50\%) are resident species, $58(40 \%)$ local migrants and $14(10 \%)$ winter migrants (Table 1). The Green Sandpiper Tringa ochropus, Indian Pitta Pitta brachyura, Forest Wagtail Dendronanthus indicus, Yellow Wagtail Motacilla flava, Brown Shrike Lanius cristatus, Blyth's Reed-Warbler Acrocephalus dumetorum, Greenish Leaf-Warbler Phylloscopus trochiloides, Hume's Lesser Whitethroat Sylvia althaea, Asian Brown Flycatcher Muscicapa dauurica, Verditer Flycatcher Eumyias thalassina, Common Rosefinch Carpodacus erythrinus, and Rosy Starling Sturnus roseus are some important winter migrants and usually seen between mid November and early January, of these, the Green Sandpiper, Indian Pitta and the Grey Wagtail are 
also seen during the summer season in the study area.

Among the total species observed, 45 (31\%) species are uncommon, 38 (26\%) common, 26 (18\%) occasional, $24(17 \%)$ rare and 12 (8\%) abundant (Table 1). The most abundant species in the study area are Red-whiskered Bulbul Pycnonotus jocosus, Red-vented Bulbul P. cafer, White-browed Bulbul P. luteolus, White-headed Babbler Turdoides affinis, Blue-winged Parakeet $P$. columboides, Small Bee-eater Merops orientalis, Common Iora Aegithina tiphia, Spotted Dove Streptopelia chinensis and Purple-rumped Sunbird Nectarinia zeylonica. The White-necked Stork Ciconia episcopus, Sirkeer Malkoha Phaenicophaeus leschenaultii, Black Bulbul Hypsipetes leucocephalus, Orange-headed Thrush Zoothera citrina and Asian Fairy-Bluebird Irena puella were recorded only once during the entire survey period. Twenty-two species of birds were observed to be restricted to the stream side vegetations including some evergreen forest bird species (Appendix 1).

Forty species were recorded breeding in and around the SACON campus (Appendix 1). Of these, 23 species were seen to be nesting while the remaining 17 species were observed with their juveniles. Most of the species started their breeding activities at the end of the northeast monsoon.

Nirmala (2002) reported 187 species of birds from a larger area in the Anaikatty Hills of Western Ghats. The present study which focussed on a subset area of the earlier study, reports 19 additional species (Appendix 1) that were not reported by Nirmala (2002). However, there were 61 species which are found in the earlier checklist that could not be observed during the present study, because the study was carried out in relatively smaller and selected areas only. We hope that this updated checklist of birds would serve as a guide to the bird-watching community especially to students and research scholars and those who visit the SACON campus.

\section{REFERENCES}

Ali, S. (2002). The Book of Indian Birds (13 ${ }^{\text {th }}$ Revised Edition). Oxford University Press, New Delhi, 326pp.

Balasubramanian, P., P. Nehru \& M.K. Sebastian (2012). Forest Genetic Resources Conservation in SACON, Anaikatty, Coimbatore, pp. 314319. In: Palanisamy, K., N. Krishnakumar \& R. Anandalakshmi (eds.). Forest Genetic Resources Management in India. Institute of Forest Genetics and Tree breeding, Coimbatore.

Blair, R.B. (1999). Birds and butterflies; surrogate taxa for assessing biodiversity? Ecological Application 9: 164-170.

Grimmett, R., C. Inskipp \& T. Inskipp (2011). Birds of the India, Pakistan, Nepal, Bangladesh, Bhutan, Sri Lanka and the Maldives. Princeton University Press, New Jersey, 528pp.

Islam, M.Z. \& A.R. Rahmani (2004). Important Bird Areas in India: Priority Sites for Conservation. Indian Bird Conservation Network: Bombay Natural History Society and Birdlife International (UK), xviii+1133pp.

Manakadan, R. \& A. Pittie (2001). Standardized Common and Scientific Names of the Birds of the Indian Subcontinent. Buceros 6(1): 1-37. Nirmala, T. (2002). Ecology of birds communities in the Anaikatty Hills, Coimbatore. PhD Thesis, Bharathiar University, Coimbatore.

Table 1. Distribution of number of species with orders, families and status of avifauna in and around the SACON campus

\begin{tabular}{|c|c|c|c|c|c|c|c|c|c|c|}
\hline \multirow{2}{*}{ Orders } & \multirow{2}{*}{$\begin{array}{l}\text { No. of } \\
\text { Families }\end{array}$} & \multirow{2}{*}{$\begin{array}{l}\text { No. of } \\
\text { Species }\end{array}$} & \multicolumn{3}{|c|}{ Migratory status } & \multicolumn{5}{|c|}{ Abundance status } \\
\hline & & & RE & LM & WM & A & C & U & 0 & $\mathbf{R}$ \\
\hline Pelicaniformes & 1 & 1 & - & 1 & - & - & - & - & 1 & - \\
\hline Ciconiiformes & 2 & 4 & 2 & 2 & - & - & - & 3 & - & 1 \\
\hline Falconiformes & 2 & 13 & 4 & 9 & - & - & - & 5 & 4 & 4 \\
\hline Galliformes & 1 & 3 & 3 & - & - & - & 3 & - & - & - \\
\hline Gruiformes & 2 & 2 & 1 & 1 & - & - & - & 1 & - & 1 \\
\hline Charadriiformes & 2 & 2 & 1 & - & 1 & - & - & 2 & - & - \\
\hline Columbiformes & 1 & 6 & 4 & 2 & - & 2 & - & 3 & 1 & - \\
\hline Psittaciformes & 1 & 4 & 2 & 2 & - & 1 & - & 2 & - & 1 \\
\hline Cuculiformes & 1 & 8 & 3 & 4 & 1 & - & 2 & 3 & 2 & 1 \\
\hline Strigiformes & 1 & 2 & 2 & - & - & - & 1 & 1 & - & - \\
\hline Caprimulgiformes & 1 & 2 & 2 & - & - & - & - & 2 & - & - \\
\hline Apodiformes & 2 & 3 & 2 & 1 & - & - & 2 & 1 & - & - \\
\hline Coraciiformes & 5 & 10 & 5 & 5 & - & 1 & 1 & 6 & 1 & 1 \\
\hline Piciformes & 2 & 9 & 4 & 5 & - & - & 3 & 2 & 3 & 1 \\
\hline Passeriformes & 24 & 76 & 38 & 26 & 12 & 8 & 26 & 14 & 14 & 14 \\
\hline Total & 48 & 145 & 73 & 58 & 14 & 12 & 38 & 45 & 26 & 24 \\
\hline
\end{tabular}

RE - Resident; LM - Local Migrant; WM - Winter Migrant; A - Abundant; C - Common; U - Uncommon; O - Occasional; R: Rare 
Appendix 1. Systematic list of birds with its status in and around the SACON campus, southern India during January 2012 to May 2013

\begin{tabular}{|c|c|c|c|c|c|}
\hline & $\begin{array}{l}\text { Family / Common } \\
\text { Name }\end{array}$ & Scientific Name & MS & AS & BR \\
\hline & Phalacrocoracidae & & & & \\
\hline \multirow[t]{2}{*}{1} & Little Cormorant^^ & $\begin{array}{l}\text { Phalacrocorax } \\
\text { niger }\end{array}$ & LM & 0 & \\
\hline & Ardeidae & & & & \\
\hline 2 & Little Egret & Egretta garzetta & $\mathrm{RE}$ & $u$ & \\
\hline 3 & Cattle Egret & Bubulcus ibis & LM & $u$ & \\
\hline \multirow[t]{2}{*}{4} & Indian Pond-Heron & Ardeola grayii & RE & U & \\
\hline & Ciconiidae & & & & \\
\hline \multirow[t]{2}{*}{5} & $\begin{array}{l}\text { White-necked } \\
\text { Stork^*}\end{array}$ & Ciconia episcopus & LM & $\mathrm{R}$ & \\
\hline & Accipitridae & & & & \\
\hline 6 & $\begin{array}{l}\text { Oriental Honey- } \\
\text { Buzzard* }\end{array}$ & $\begin{array}{l}\text { Pernis } \\
\text { ptilorhynchus }\end{array}$ & LM & U & \\
\hline 7 & Black-shouldered Kite & Elanus caeruleus & LM & U & \\
\hline 8 & Black Kite & Milvus migrans & RE & $\mathrm{O}$ & \\
\hline 9 & Brahminy Kite & Haliastur indus & $\mathrm{RE}$ & 0 & \\
\hline 10 & 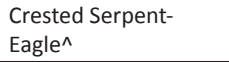 & Spilornis cheela & LM & 0 & \\
\hline 11 & Shikra & Accipiter badius & $\mathrm{RE}$ & $u$ & \\
\hline 12 & $\begin{array}{l}\text { Besra } \\
\text { Sparrowhawk }{ }^{\wedge}\end{array}$ & Accipiter virgatus & LM & $R$ & \\
\hline 13 & White-eyed Buzzard* & Butastur teesa & LM & 0 & \\
\hline 14 & Black Eagle & $\begin{array}{l}\text { Ictinaetus } \\
\text { malayensis }\end{array}$ & RE & $u$ & \\
\hline 15 & Bonelli's Eagle* & $\begin{array}{l}\text { Hieraaetus } \\
\text { fasciatus }\end{array}$ & LM & $u$ & \\
\hline 16 & Rufous-bellied Eagle* & Hieraaetus kienerii & LM & $\mathrm{R}$ & \\
\hline \multirow[t]{2}{*}{17} & $\begin{array}{l}\text { Changeable Hawk- } \\
\text { Eagle^}^{\wedge}\end{array}$ & Spizaetus cirrhatus & LM & $\mathrm{R}$ & \\
\hline & Falconidae & & & & \\
\hline \multirow[t]{2}{*}{18} & Common Kestrel* & Falco tinnunculus & LM & $\mathrm{R}$ & \\
\hline & Phasianidae & & & & \\
\hline 19 & Grey Francolin & $\begin{array}{l}\text { Francolinus } \\
\text { pondicerianus }\end{array}$ & RE & C & $\#$ \\
\hline 20 & Grey Junglefowl & Gallus sonneratii & RE & C & $\#$ \\
\hline \multirow[t]{2}{*}{21} & Indian Peafowl & Pavo cristatus & $\mathrm{RE}$ & C & $\#$ \\
\hline & Turnicidae & & & & \\
\hline \multirow[t]{2}{*}{22} & Common Buttonquail & Turnix suscitator & LM & $\mathrm{R}$ & $\$$ \\
\hline & Rallidae & & & & \\
\hline \multirow[t]{2}{*}{23} & $\begin{array}{l}\text { White-breasted } \\
\text { Waterhen }\end{array}$ & $\begin{array}{l}\text { Amaurornis } \\
\text { phoenicurus }\end{array}$ & $\mathrm{RE}$ & $u$ & \\
\hline & Charadriidae & & & & \\
\hline \multirow[t]{2}{*}{24} & Red-wattled Lapwing & Vanellus indicus & RE & $u$ & $\$$ \\
\hline & Scolopacidae & & & & \\
\hline \multirow[t]{2}{*}{25} & Green Sandpiper^* & Tringa ochropus & WM & $u$ & \\
\hline & Columbidae & & & & \\
\hline 26 & Blue Rock Pigeon & Columba livia & RE & $u$ & \\
\hline 27 & Little Brown Dove & $\begin{array}{l}\text { Streptopelia } \\
\text { senegalensis }\end{array}$ & RE & A & \\
\hline 28 & Spotted Dove & $\begin{array}{l}\text { Streptopelia } \\
\text { chinensis }\end{array}$ & $\mathrm{RE}$ & A & $\$$ \\
\hline
\end{tabular}

\begin{tabular}{|c|c|c|c|c|c|}
\hline & $\begin{array}{l}\text { Family / Common } \\
\text { Name }\end{array}$ & Scientific Name & MS & AS & BR \\
\hline 29 & $\begin{array}{l}\text { Eurasian Collared- } \\
\text { Dove }\end{array}$ & $\begin{array}{l}\text { Streptopelia } \\
\text { decaocto }\end{array}$ & RE & $U$ & \\
\hline 30 & Emerald Dove & Chalcophaps indica & LM & $U$ & \\
\hline \multirow[t]{2}{*}{31} & $\begin{array}{l}\text { Yellow-legged Green- } \\
\text { Pigeon }\end{array}$ & $\begin{array}{l}\text { Treron } \\
\text { phoenicoptera }\end{array}$ & LM & $\mathrm{O}$ & \\
\hline & Psittacidae & & & & \\
\hline 32 & $\begin{array}{l}\text { Indian Hanging- } \\
\text { Parrot }\end{array}$ & Loriculus vernalis & LM & $\mathrm{R}$ & \\
\hline 33 & Rose-ringed Parakeet & Psittacula krameri & LM & $U$ & \\
\hline 34 & $\begin{array}{l}\text { Plum-headed } \\
\text { Parakeet }\end{array}$ & $\begin{array}{l}\text { Psittacula } \\
\text { cyanocephala }\end{array}$ & RE & $U$ & \\
\hline \multirow[t]{2}{*}{35} & Blue-winged Parakeet & $\begin{array}{l}\text { Psittacula } \\
\text { columboides }\end{array}$ & RE & A & $\#$ \\
\hline & Cuculidae & & & & \\
\hline 36 & Pied Crested Cuckoo & Clamator jacobinus & LM & $U$ & \\
\hline 37 & Brainfever Bird & Hierococcyx varius & LM & $U$ & \\
\hline 38 & Common Cuckoo & Cuculus canorus & WM & 0 & \\
\hline 39 & $\begin{array}{l}\text { Indian Plaintive } \\
\text { Cuckoo* }\end{array}$ & $\begin{array}{l}\text { Cacomantis } \\
\text { passerinus }\end{array}$ & LM & $\mathrm{O}$ & \\
\hline 40 & Asian Koel & $\begin{array}{l}\text { Eudynamys } \\
\text { scolopacea }\end{array}$ & RE & $\mathrm{C}$ & $\#$ \\
\hline 41 & $\begin{array}{l}\text { Small Green-billed } \\
\text { Malkoha }\end{array}$ & $\begin{array}{l}\text { Phaenicophaeus } \\
\text { viridirostris }\end{array}$ & RE & $\mathrm{C}$ & $\#$ \\
\hline 42 & Sirkeer Malkoha & $\begin{array}{l}\text { Phaenicophaeus } \\
\text { leschenaultii }\end{array}$ & LM & $\mathrm{R}$ & \\
\hline \multirow[t]{2}{*}{43} & Greater Coucal & Centropus sinensis & RE & $U$ & $\#$ \\
\hline & Strigidae & & & & \\
\hline 44 & Brown Fish-Owl & Ketupa zeylonensis & RE & $U$ & $\#$ \\
\hline \multirow[t]{2}{*}{45} & Spotted Owlet & Athene brama & RE & C & $\$$ \\
\hline & Caprimulgidae & & & & \\
\hline 46 & Jerdon's Nightjar* & $\begin{array}{l}\text { Caprimulgus } \\
\text { atripennis }\end{array}$ & RE & $U$ & \\
\hline \multirow[t]{2}{*}{47} & $\begin{array}{l}\text { Common Indian } \\
\text { Nightjar }\end{array}$ & $\begin{array}{l}\text { Caprimulgus } \\
\text { asiaticus }\end{array}$ & RE & $U$ & \\
\hline & Apodidae & & & & \\
\hline 48 & Asian Palm-Swift & $\begin{array}{l}\text { Cypsiurus } \\
\text { balasiensis }\end{array}$ & RE & $\mathrm{C}$ & \\
\hline \multirow[t]{2}{*}{49} & House Swift & Apus affinis & RE & C & \\
\hline & Hemiprocnidae & & & & \\
\hline \multirow[t]{2}{*}{50} & Crested Tree-Swift & $\begin{array}{l}\text { Hemiprocne } \\
\text { coronata }\end{array}$ & LM & $U$ & \\
\hline & Alcedinidae & & & & \\
\hline 51 & Small Blue Kingfisher & Alcedo atthis & RE & $U$ & \\
\hline 52 & $\begin{array}{l}\text { Stork-billed } \\
\text { Kingfisher^*}\end{array}$ & Halcyon capensis & LM & $\mathrm{R}$ & \\
\hline 53 & $\begin{array}{l}\text { White-breasted } \\
\text { Kingfisher }\end{array}$ & $\begin{array}{l}\text { Halcyon } \\
\text { smyrnensis }\end{array}$ & RE & $U$ & $\#$ \\
\hline \multirow[t]{2}{*}{54} & Lesser Pied Kingfisher & Ceryle rudis & LM & $U$ & \\
\hline & Meropidae & & & & \\
\hline 55 & $\begin{array}{l}\text { Blue-bearded Bee- } \\
\text { eater }\end{array}$ & $\begin{array}{l}\text { Nyctyornis } \\
\text { athertoni }\end{array}$ & LM & U & \\
\hline 56 & Small Bee-eater & Merops orientalis & RE & A & $\#$ \\
\hline 57 & $\begin{array}{l}\text { Chestnut-headed } \\
\text { Bee-eater }\end{array}$ & $\begin{array}{l}\text { Merops } \\
\text { leschenaulti }\end{array}$ & LM & U & \\
\hline
\end{tabular}




\begin{tabular}{|c|c|c|c|c|c|}
\hline & $\begin{array}{l}\text { Family / Common } \\
\text { Name }\end{array}$ & Scientific Name & MS & AS & BR \\
\hline & Coraciidae & & & & \\
\hline \multirow[t]{2}{*}{58} & Indian Roller & $\begin{array}{l}\text { Coracias } \\
\text { benghalensis }\end{array}$ & RE & $U$ & \\
\hline & Upupidae & & & & \\
\hline \multirow[t]{2}{*}{59} & Common Hoopoe & Upupa epops & RE & C & $\$$ \\
\hline & Bucerotidae & & & & \\
\hline \multirow[t]{2}{*}{60} & Indian Grey Hornbill & Ocyceros birostris & LM & 0 & \\
\hline & Capitonidae & & & & \\
\hline 61 & $\begin{array}{l}\text { Brown-headed } \\
\text { Barbet }\end{array}$ & $\begin{array}{l}\text { Megalaima } \\
\text { zeylanica }\end{array}$ & LM & 0 & \\
\hline 62 & $\begin{array}{l}\text { White-cheeked } \\
\text { Barbet }\end{array}$ & Megalaima viridis & LM & 0 & \\
\hline \multirow[t]{2}{*}{63} & Coppersmith Barbet & $\begin{array}{l}\text { Megalaima } \\
\text { haemacephala }\end{array}$ & RE & C & $\$$ \\
\hline & Picidae & & & & \\
\hline 64 & $\begin{array}{l}\text { Brown-capped Pygmy } \\
\text { Woodpecker^^}\end{array}$ & $\begin{array}{l}\text { Dendrocopos } \\
\text { nanus }\end{array}$ & LM & $\mathrm{R}$ & \\
\hline 65 & Rufous Woodpecker & Celeus brachyurus & LM & 0 & \\
\hline 66 & $\begin{array}{l}\text { Small Yellow-naped } \\
\text { Woodpecker }\end{array}$ & Picus chlorolophus & RE & $U$ & \\
\hline 67 & $\begin{array}{l}\text { Little Scaly-bellied } \\
\text { Green Woodpecker }\end{array}$ & $\begin{array}{l}\text { Picus } \\
\text { xanthopygaeus }\end{array}$ & RE & $C$ & \\
\hline 68 & $\begin{array}{l}\text { Common Golden- } \\
\text { backed Woodpecker }\end{array}$ & $\begin{array}{l}\text { Dinopium } \\
\text { javanense }\end{array}$ & LM & $U$ & \\
\hline \multirow[t]{2}{*}{69} & $\begin{array}{l}\text { Lesser Golden- } \\
\text { backed Woodpecker }\end{array}$ & $\begin{array}{l}\text { Dinopium } \\
\text { benghalense }\end{array}$ & RE & C & $\$$ \\
\hline & Pittidae & & & & \\
\hline \multirow[t]{2}{*}{70} & Indian Pitta & Pitta brachyura & WM & 0 & \\
\hline & Alaudidae & & & & \\
\hline 71 & Jerdon' s Bush-Lark* & Mirafra affinis & $\mathrm{RE}$ & $u$ & \\
\hline \multirow[t]{2}{*}{72} & Eastern Skylark* & Alauda gulgula & RE & $U$ & \\
\hline & Hirundinidae & & & & \\
\hline 73 & Common Swallow & Hirundo rustica & LM & $\mathrm{R}$ & \\
\hline \multirow[t]{2}{*}{74} & Red-rumped Swallow & Hirundo daurica & LM & $u$ & \\
\hline & Motacillidae & & & & \\
\hline 75 & Forest Wagtail^ & $\begin{array}{l}\text { Dendronanthus } \\
\text { indicus }\end{array}$ & WM & 0 & \\
\hline 76 & Large Pied Wagtail & $\begin{array}{l}\text { Motacilla } \\
\text { maderaspatensis }\end{array}$ & RE & $U$ & \\
\hline 77 & Yellow Wagtail & Motacilla flava & WM & $\mathrm{R}$ & \\
\hline \multirow[t]{2}{*}{78} & Grey Wagtail^ & Motacilla cinerea & WM & 0 & \\
\hline & Campephagidae & & & & \\
\hline 79 & $\begin{array}{l}\text { Black-headed } \\
\text { Cuckoo-Shrike }\end{array}$ & $\begin{array}{l}\text { Coracina } \\
\text { melanoptera }\end{array}$ & LM & 0 & \\
\hline 80 & Small Minivet & $\begin{array}{l}\text { Pericrocotus } \\
\text { cinnamomeus }\end{array}$ & RE & $\mathrm{C}$ & $\#$ \\
\hline \multirow[t]{2}{*}{81} & Common Woodshrike & $\begin{array}{l}\text { Tephrodornis } \\
\text { pondicerianus }\end{array}$ & RE & $\mathrm{C}$ & \\
\hline & Pycnonotidae & & & & \\
\hline 82 & Red-whiskered Bulbul & Pycnonotus jocosus & RE & A & $\$$ \\
\hline 83 & Red-vented Bulbul & Pycnonotus cafer & RE & $A$ & $\$$ \\
\hline 84 & White-browed Bulbul & $\begin{array}{l}\text { Pycnonotus } \\
\text { luteolus }\end{array}$ & RE & A & $\$$ \\
\hline 85 & Black Bulbul^^ & $\begin{array}{l}\text { Hypsipetes } \\
\text { leucocephalus }\end{array}$ & LM & $\mathrm{R}$ & \\
\hline
\end{tabular}

\begin{tabular}{|c|c|c|c|c|c|}
\hline & $\begin{array}{l}\text { Family / Common } \\
\text { Name }\end{array}$ & Scientific Name & MS & AS & BR \\
\hline & Irenidae & & & & \\
\hline 86 & Common lora & Aegithina tiphia & RE & $A$ & $\$$ \\
\hline 87 & Jerdon's Chloropsis & $\begin{array}{l}\text { Chloropsis } \\
\text { cochinchinensis }\end{array}$ & RE & $A$ & \\
\hline 88 & $\begin{array}{l}\text { Gold-fronted } \\
\text { Chloropsis }\end{array}$ & $\begin{array}{l}\text { Chloropsis } \\
\text { aurifrons }\end{array}$ & RE & $A$ & \\
\hline \multirow[t]{2}{*}{89} & $\begin{array}{l}\text { Asian Fairy- } \\
\text { Bluebird^*}\end{array}$ & Irena puella & LM & $\mathrm{R}$ & \\
\hline & Laniidae & & & & \\
\hline 90 & Brown Shrike & Lanius cristatus & WM & $\mathrm{R}$ & \\
\hline \multirow[t]{2}{*}{91} & Rufous-backed Shrike & Lanius schach & LM & $\mathrm{R}$ & \\
\hline & Turdinae & & & & \\
\hline 92 & $\begin{array}{l}\text { Malabar Whistling- } \\
\text { Thrush^ }\end{array}$ & $\begin{array}{l}\text { Myiophonus } \\
\text { horsfieldii }\end{array}$ & LM & $\mathrm{R}$ & \\
\hline 93 & $\begin{array}{l}\text { Orange-headed } \\
\text { Thrush^*}\end{array}$ & Zoothera citrina & LM & $\mathrm{R}$ & \\
\hline 94 & Eurasian Blackbird^* & Turdus merula & LM & $\mathrm{R}$ & \\
\hline 95 & $\begin{array}{l}\text { Oriental Magpie- } \\
\text { Robin }\end{array}$ & Copsychus saularis & RE & $C$ & $\#$ \\
\hline 96 & Indian Robin & $\begin{array}{l}\text { Saxicoloides } \\
\text { fulicata }\end{array}$ & RE & C & $\$$ \\
\hline \multirow[t]{2}{*}{97} & Pied Bushchat & Saxicola caprata & RE & $C$ & $\#$ \\
\hline & Timaliinae & & & & \\
\hline 98 & Spotted Babbler^ & $\begin{array}{l}\text { Pellorneum } \\
\text { ruficeps }\end{array}$ & LM & 0 & \\
\hline 99 & $\begin{array}{l}\text { Indian Scimitar- } \\
\text { Babbler^ }\end{array}$ & $\begin{array}{l}\text { Pomatorhinus } \\
\text { horsfieldii }\end{array}$ & LM & $\mathrm{R}$ & \\
\hline 100 & $\begin{array}{l}\text { Rufous-bellied } \\
\text { Babbler }\end{array}$ & $\begin{array}{l}\text { Dumetia } \\
\text { hyperythra }\end{array}$ & RE & $C$ & $\$$ \\
\hline 101 & Yellow-eyed Babbler & $\begin{array}{l}\text { Chrysomma } \\
\text { sinense }\end{array}$ & RE & $C$ & $\$$ \\
\hline 102 & Large Grey Babbler* & Turdoides malcolmi & RE & $C$ & \\
\hline 103 & Jungle Babbler & Turdoides striatus & $\mathrm{RE}$ & $U$ & \\
\hline \multirow[t]{2}{*}{104} & $\begin{array}{l}\text { White-headed } \\
\text { Babbler }\end{array}$ & Turdoides affinis & RE & $A$ & $\$$ \\
\hline & Sylviinae & & & & \\
\hline 105 & Franklin's Prinia & Prinia hodgsonii & LM & $U$ & \\
\hline 106 & Jungle Prinia & Prinia sylvatica & LM & $U$ & \\
\hline 107 & Ashy Prinia & Prinia socialis & $\mathrm{RE}$ & $c$ & $\$$ \\
\hline 108 & Plain Prinia & Prinia inornata & LM & $U$ & \\
\hline 109 & Blyth's Reed-Warbler & $\begin{array}{l}\text { Acrocephalus } \\
\text { dumetorum }\end{array}$ & WM & $\mathrm{R}$ & \\
\hline 110 & Booted Warbler & Hippolais caligata & RE & C & \\
\hline 111 & Common Tailorbird & $\begin{array}{l}\text { Orthotomus } \\
\text { sutorius }\end{array}$ & RE & $C$ & $\$$ \\
\hline 112 & $\begin{array}{l}\text { Greenish Leaf- } \\
\text { Warbler }\end{array}$ & $\begin{array}{l}\text { Phylloscopus } \\
\text { trochiloides }\end{array}$ & WM & $C$ & \\
\hline \multirow[t]{2}{*}{113} & $\begin{array}{l}\text { Hume's Lesser } \\
\text { Whitethroat }\end{array}$ & Sylvia althaea & WM & 0 & \\
\hline & Muscicapinae & & & & \\
\hline 114 & $\begin{array}{l}\text { Asian Brown } \\
\text { Flycatcher }\end{array}$ & $\begin{array}{l}\text { Muscicapa } \\
\text { dauurica }\end{array}$ & WM & 0 & \\
\hline 115 & Verditer Flycatcher & Eumyias thalassina & WM & 0 & \\
\hline 116 & $\begin{array}{l}\text { Tickell's Blue- } \\
\text { Flycatcher^ }^{\wedge}\end{array}$ & Cyornis tickelliae & RE & $U$ & \\
\hline
\end{tabular}




\begin{tabular}{|c|c|c|c|c|c|}
\hline & $\begin{array}{l}\text { Family / Common } \\
\text { Name }\end{array}$ & Scientific Name & MS & AS & BR \\
\hline & Monarchinae & & & & \\
\hline 117 & $\begin{array}{l}\text { Asian Paradise- } \\
\text { Flycatcher }\end{array}$ & $\begin{array}{l}\text { Terpsiphone } \\
\text { paradisi }\end{array}$ & $\mathrm{RE}$ & C & \\
\hline \multirow[t]{2}{*}{118} & $\begin{array}{l}\text { Black-naped } \\
\text { Monarch-Flycatcher* }\end{array}$ & Hypothymis azurea & LM & $\mathrm{R}$ & \\
\hline & Paridae & & & & \\
\hline \multirow[t]{2}{*}{119} & Great Tit & Parus major & RE & C & \\
\hline & Dicaeidae & & & & \\
\hline \multirow[t]{2}{*}{120} & Tickell's Flowerpecker & $\begin{array}{l}\text { Dicaeum } \\
\text { erythrorhynchos }\end{array}$ & RE & C & \\
\hline & Nectariniidae & & & & \\
\hline 121 & $\begin{array}{l}\text { Purple-rumped } \\
\text { Sunbird }\end{array}$ & $\begin{array}{l}\text { Nectarinia } \\
\text { zeylonica }\end{array}$ & $\mathrm{RE}$ & A & $\$$ \\
\hline 122 & Purple Sunbird & Nectarinia asiatica & $\mathrm{RE}$ & C & \\
\hline \multirow[t]{2}{*}{123} & Loten's Sunbird & Nectarinia lotenia & RE & C & \\
\hline & Fringillidae & & & & \\
\hline 124 & Common Rosefinch & $\begin{array}{l}\text { Carpodacus } \\
\text { erythrinus }\end{array}$ & WM & $\mathrm{R}$ & \\
\hline 125 & $\begin{array}{l}\text { White-throated } \\
\text { Munia }\end{array}$ & $\begin{array}{l}\text { Lonchura } \\
\text { malabarica }\end{array}$ & LM & $U$ & \\
\hline 126 & $\begin{array}{l}\text { White-rumped } \\
\text { Munia* }\end{array}$ & Lonchura striata & $\mathrm{RE}$ & $U$ & \\
\hline 127 & Spotted Munia^^ & $\begin{array}{l}\text { Lonchura } \\
\text { punctulata }\end{array}$ & LM & $\mathrm{O}$ & \\
\hline \multirow[t]{2}{*}{128} & Black-headed Munia & Lonchura malacca & LM & $U$ & $\$$ \\
\hline & Passerinae & & & & \\
\hline \multirow[t]{2}{*}{129} & House Sparrow & Passer domesticus & $\mathrm{RE}$ & C & $\$$ \\
\hline & Ploceinae & & & & \\
\hline 130 & Baya Weaver & Ploceus philippinus & LM & 0 & \\
\hline
\end{tabular}

\begin{tabular}{|c|c|c|c|c|c|}
\hline & $\begin{array}{l}\text { Family / Common } \\
\text { Name }\end{array}$ & Scientific Name & MS & AS & BR \\
\hline & Sturnidae & & & & \\
\hline 131 & Brahminy Starling & $\begin{array}{l}\text { Sturnus } \\
\text { pagodarum }\end{array}$ & LM & $\mathrm{C}$ & \\
\hline 132 & Rosy Starling & Sturnus roseus & WM & 0 & \\
\hline 133 & Common Myna & Acridotheres tristis & RE & $C$ & $\$$ \\
\hline \multirow[t]{2}{*}{134} & Jungle Myna & Acridotheres fuscus & $\mathrm{RE}$ & $C$ & $\#$ \\
\hline & Oriolidae & & & & \\
\hline 135 & $\begin{array}{l}\text { Eurasian Golden } \\
\text { Oriole }\end{array}$ & Oriolus oriolus & LM & 0 & \\
\hline \multirow[t]{2}{*}{136} & Black-headed Oriole & Oriolus xanthornus & RE & C & $\#$ \\
\hline & Dicruridae & & & & \\
\hline 137 & Black Drongo & $\begin{array}{l}\text { Dicrurus } \\
\text { macrocercus }\end{array}$ & RE & C & $\#$ \\
\hline 138 & Ashy Drongo & $\begin{array}{l}\text { Dicrurus } \\
\text { leucophaeus }\end{array}$ & LM & $U$ & \\
\hline 139 & White-bellied Drongo & $\begin{array}{l}\text { Dicrurus } \\
\text { caerulescens }\end{array}$ & LM & $U$ & \\
\hline 140 & Bronzed Drongo^ & Dicrurus aeneus & LM & 0 & \\
\hline \multirow[t]{2}{*}{141} & 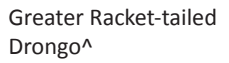 & $\begin{array}{l}\text { Dicrurus } \\
\text { paradiseus }\end{array}$ & LM & 0 & \\
\hline & Artamidae & & & & \\
\hline \multirow[t]{2}{*}{142} & Ashy Woodswallow^ & Artamus fuscus & LM & $\mathrm{R}$ & \\
\hline & Corvidae & & & & \\
\hline 143 & Indian Treepie & $\begin{array}{l}\text { Dendrocitta } \\
\text { vagabunda }\end{array}$ & RE & C & \# \\
\hline 144 & House Crow & Corvus splendens & $\mathrm{RE}$ & C & $\$$ \\
\hline 145 & Jungle Crow & $\begin{array}{l}\text { Corvus } \\
\text { macrorhynchos }\end{array}$ & RE & C & $\$$ \\
\hline
\end{tabular}

$\wedge$ - Species recorded only along the stream; * Additional sightings. These birds were sighted during the current study and were not reported by Nirmala (2002) MS - Migratory status; LM - Local migrant; RE - Resident; WM - Winter migrant; AS - Abundance status; O - Occasional; U - Uncommon; R - Rare; C - Common; A - abundant; BR - Breeding; \# - Nest-site ; \$ - Only juveniles

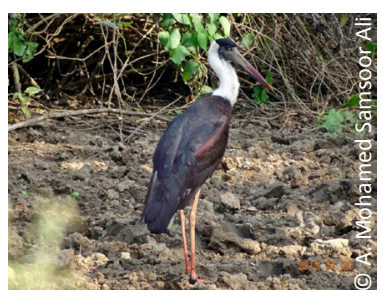

Image 1. White-necked Stork Ciconia episcopus

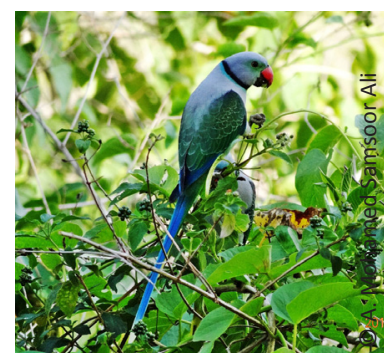

Image 5. Blue-winged Parakeet Psittacula columboides

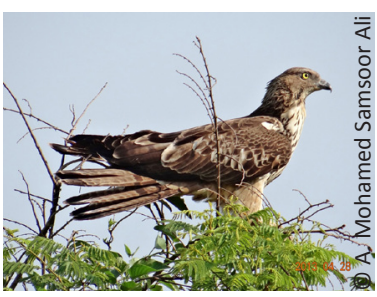

Image 2. Bonelli's Eagle

Hieraaetus fasciatus

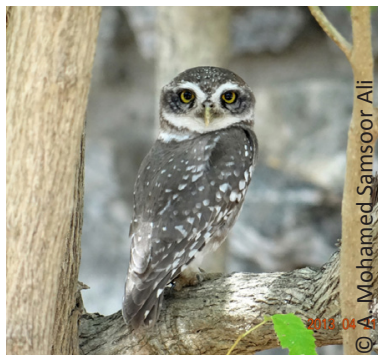

Image 6. Spotted Owlet Athene brama

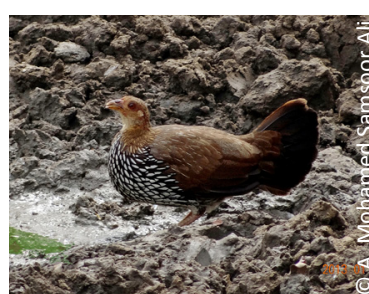

Image 3. Grey Jungle-fowl Gallus sonneratii (female)

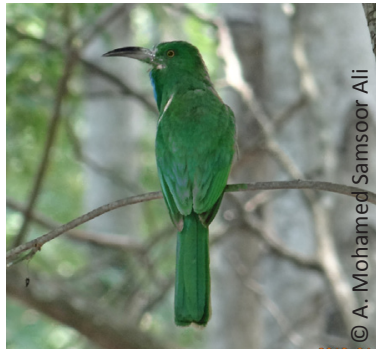

Image 7. Blue-beared Bee-eater Nyctyornis athertoni

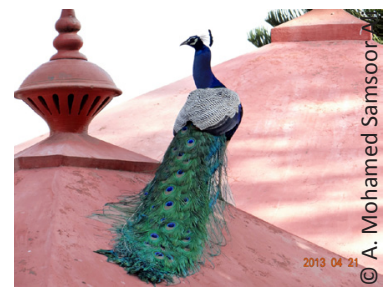

Image 4. Indian Peafowl Pavo cristatus

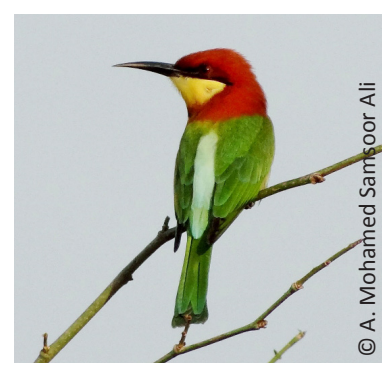

Image 8. Chestnut-headed Beeeater Merops leschenaulti 


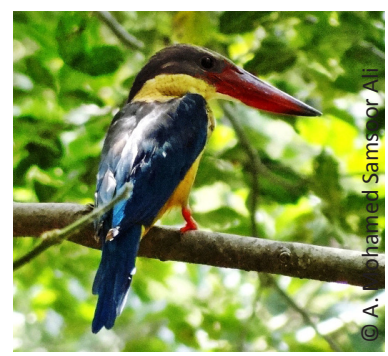

Image 9. Stork-billed Kingfisher Halcyon capensis

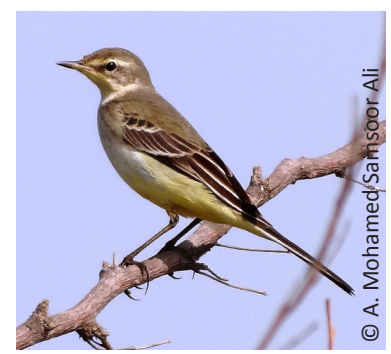

Image 13. Yellow Wagtail

Motacilla flava

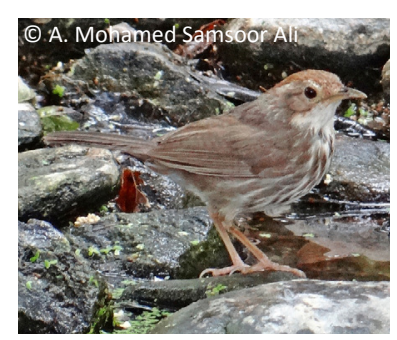

Image 17. Spotted Babbler Pellorneum ruficeps

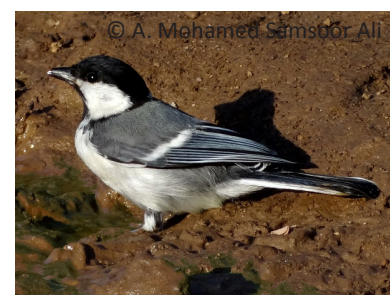

Image 21. Great Tit Parus major

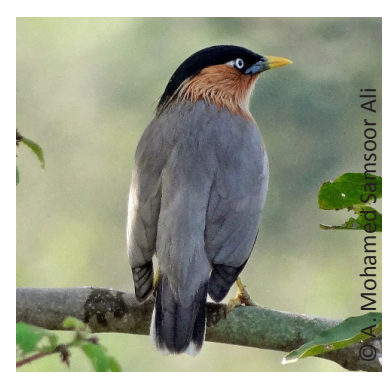

Image 22. Brahminy Starling Sturnus pagodarum

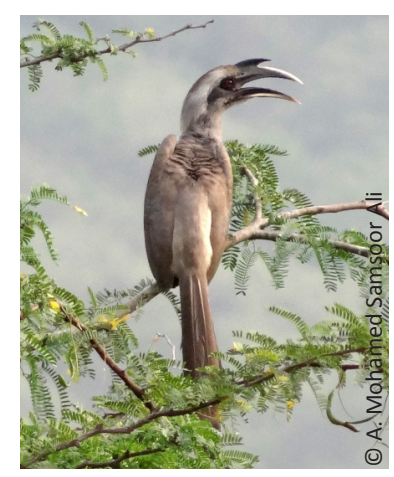

Image 10. Indian Grey Hornbill Ocyceros birostris

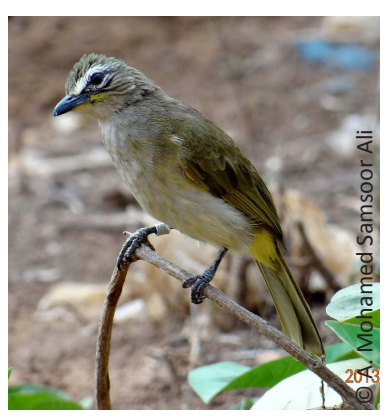

Image 14. White-browed Bulbul Pycnonotus luteolus

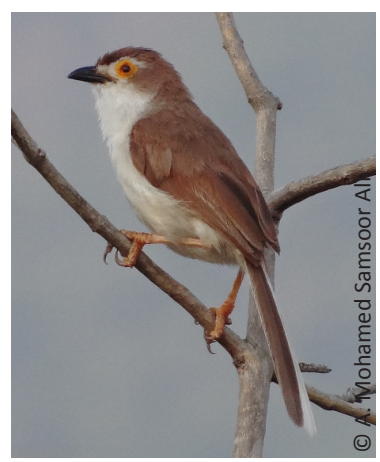

Image 18. Yellow-eyed Babbler Chrysomma sinense

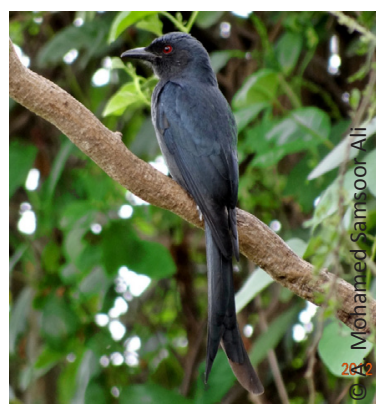

Image 23. Ashy Drongo Dicrurus leucophaeus

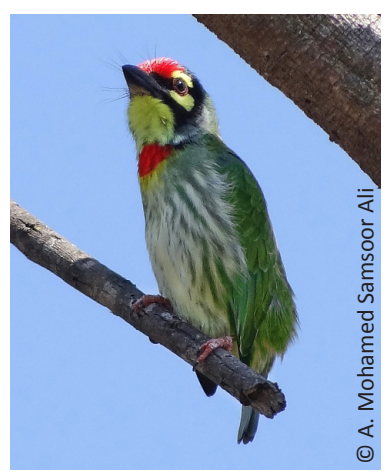

Image 11. Coppersmith Barbet Megalaima haemacephala

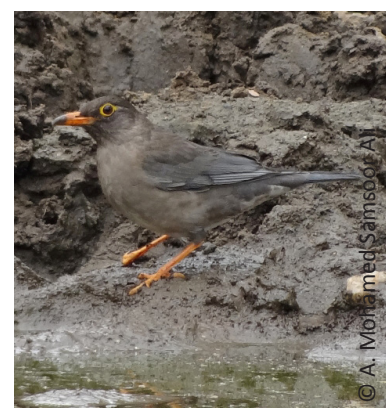

Image 15. Eurasian Blackbird Turdus merula

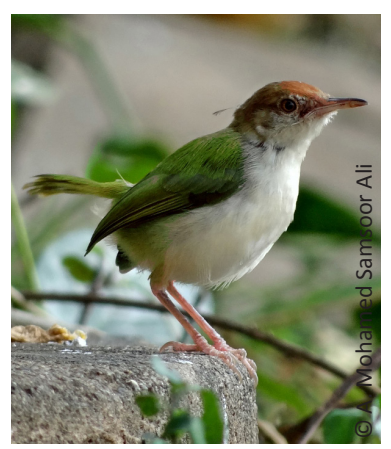

Image 19. Common Tailorbird Orthotomus sutorius

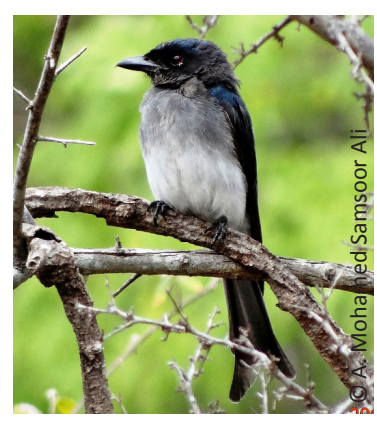

Image 24. White-bellied Drongo Dicrurus caerulescens

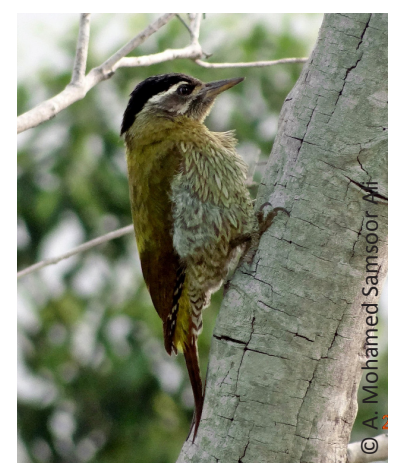

Image 12. Little Scaly-bellied Green Woodpecker Picus xanthopygaeus

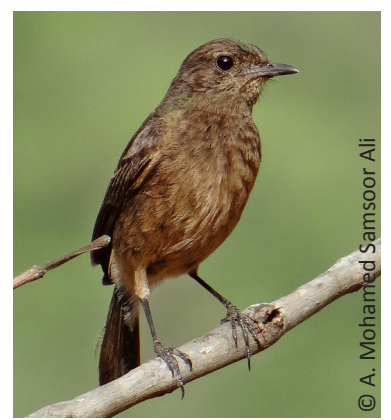

Image 16. Pied Bushchat Saxicola caprata

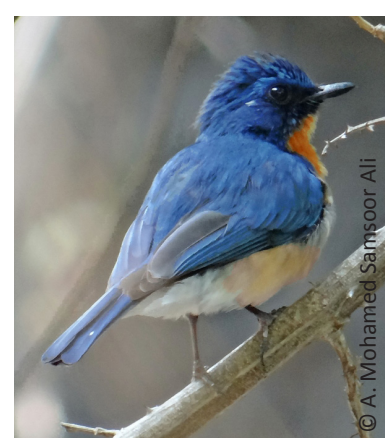

Image 20. Tickell's Blue-Flycatcher Cyornis tickelliae

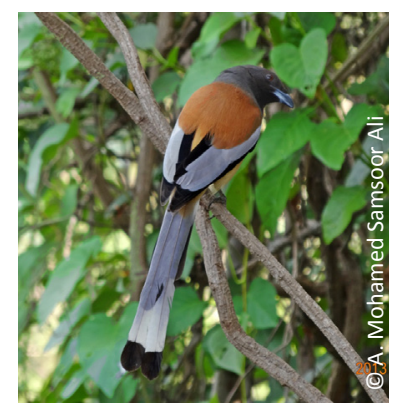

Image 25. Indian Treepie Dendrocitta vagabunda 


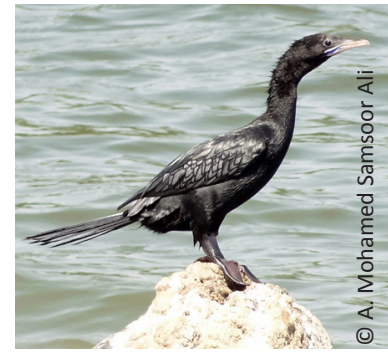

Image 26. Little Cormorant Phalacrocorax niger

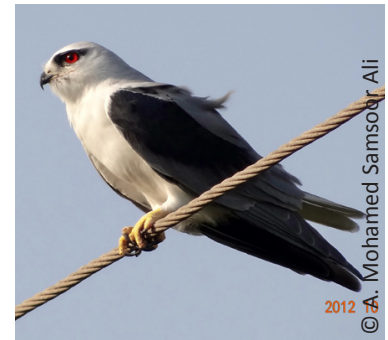

Image 30. Black-shouldered Kite Elanus caeruleus

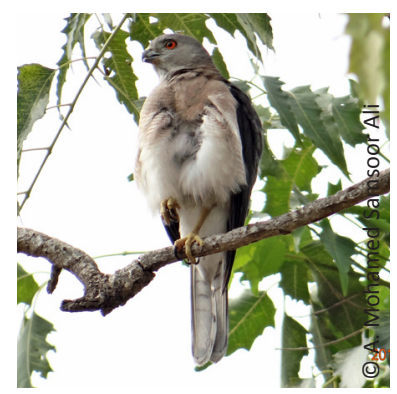

Image 34. Shikra Accipiter badius

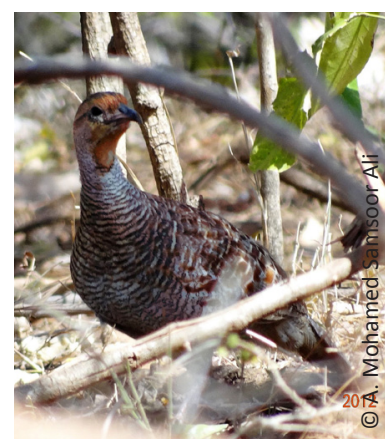

Image 38. Grey Francolin Francolinus pondicerianus
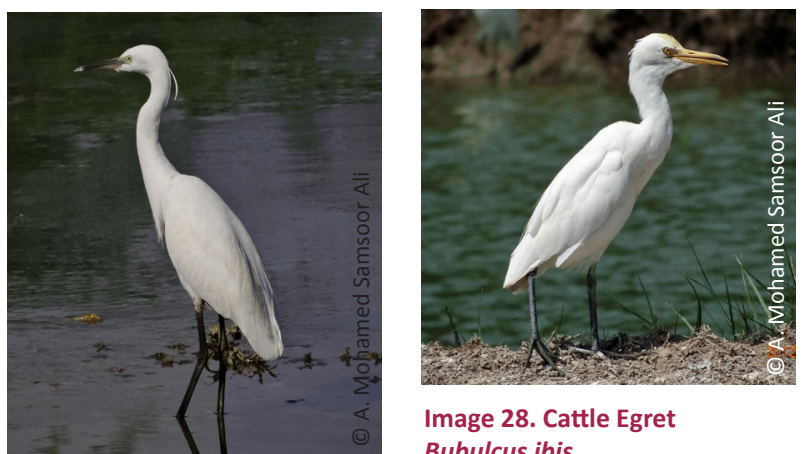

Image 28. Cattle Egret

Bubulcus ibis

Image 27. Little Egret Egretta garzetta

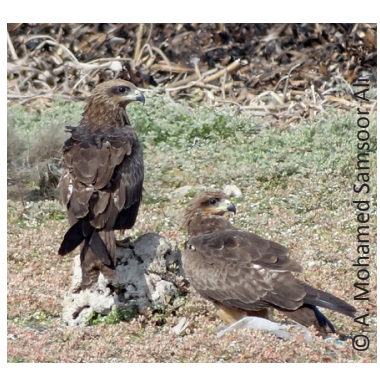

Image 31. Black Kite

Milvus migrans
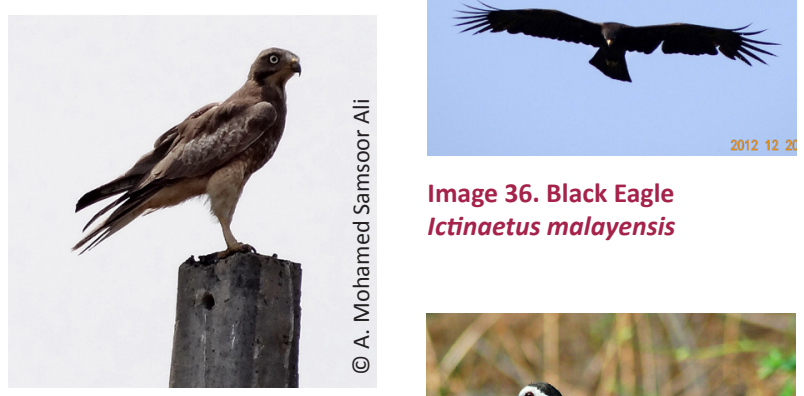

Image 36. Black Eagle Ictinaetus malayensis

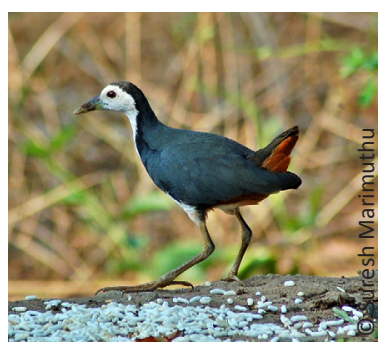
Image 40. White-breasted Waterhen Amaurornis phoenicurus

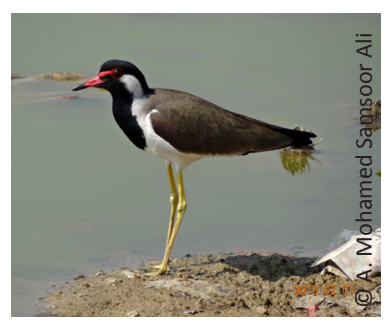

Image 41. Red-wattled Lapwing Vanellus indicus

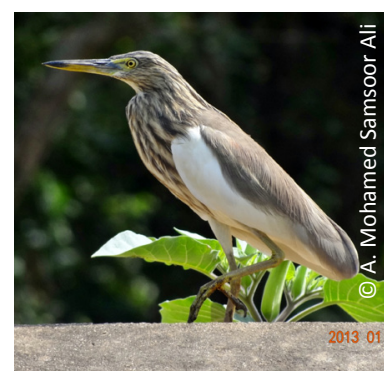

Image 29. Indian Pond-Heron Ardeola grayii

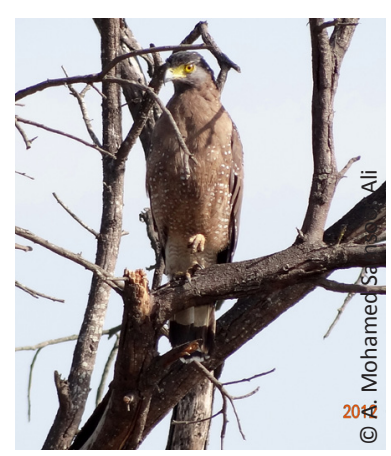

Image 33. Crested Serpent-Eagle Spilornis cheela

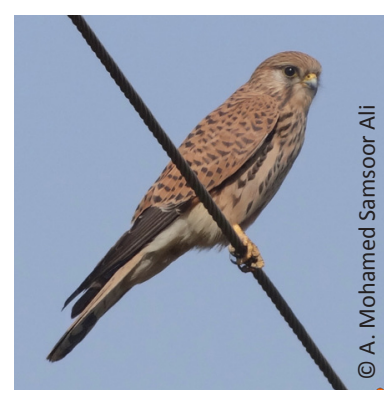

Image 37. Common Kestrel Falco tinnunculus

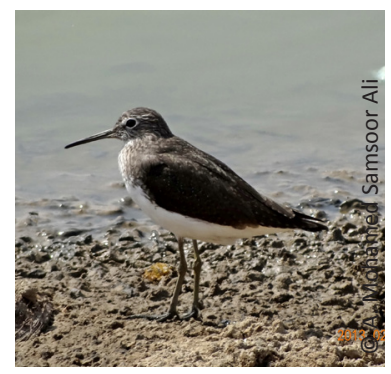

Image 42. Green Sandpiper Tringa ochropus 


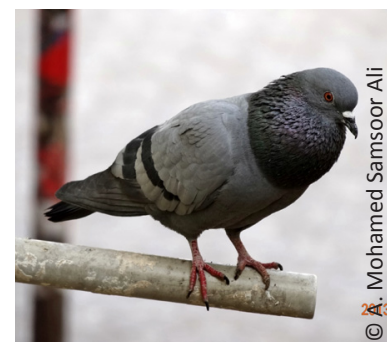

Image 43. Blue Rock Pigeon Columba livia

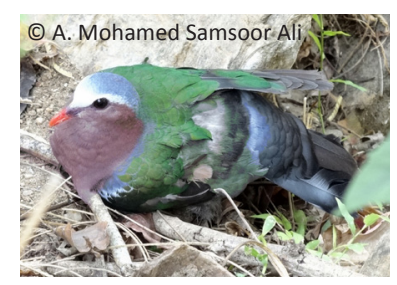

Image 47. Emerald Dove

Chalcophaps indica

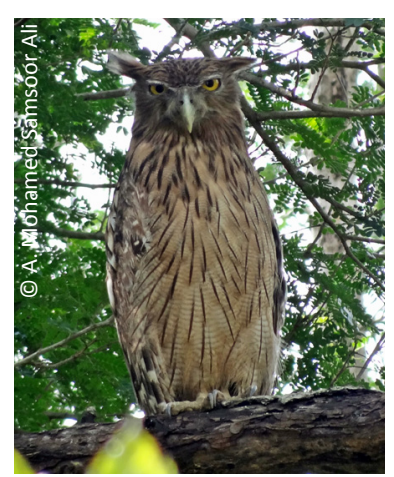

Image 51. Brown Fish-Owl Ketupa zeylonensis

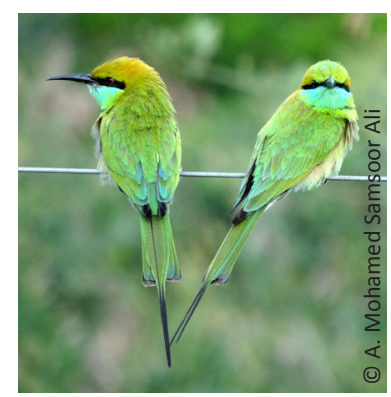

Image 55. Small Bee-eater Merops orientalis

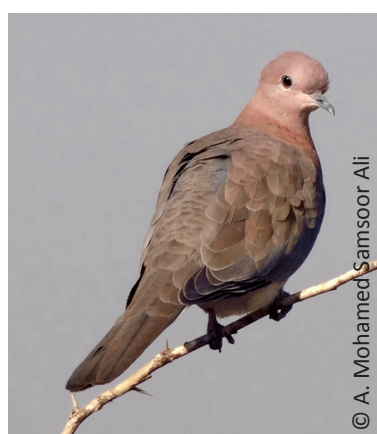

Image 44. Little Brown Dove Streptopelia senegalensis

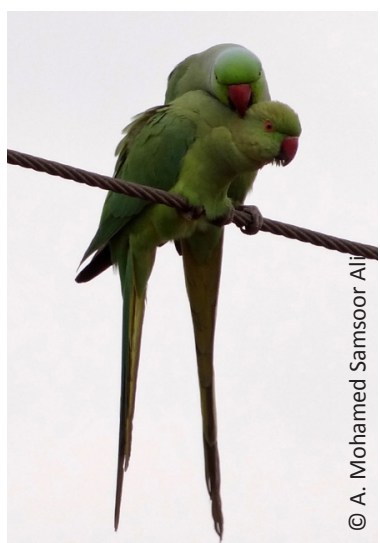

Image 48. Rose-ringed Parakeet Psittacula krameri

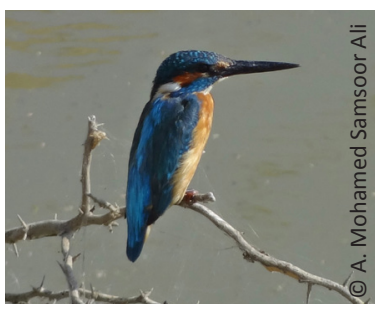

Image 52. Small Blue Kingfisher Alcedo atthis

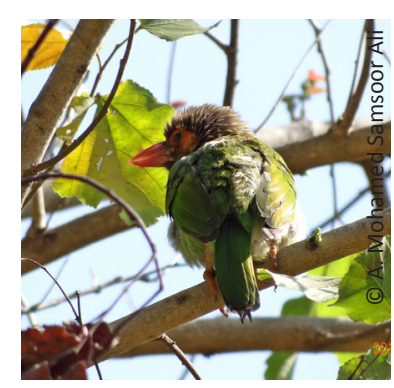

Image 56. Brown-headed Barbet Megalaima zeylanica

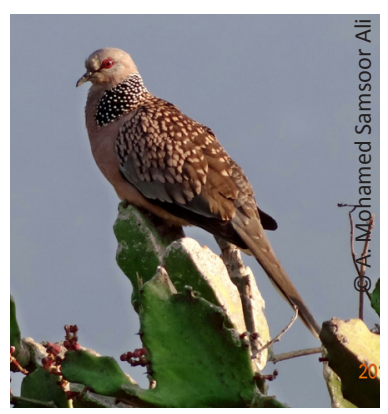

Image 45. Spotted Dove Streptopelia chinensis

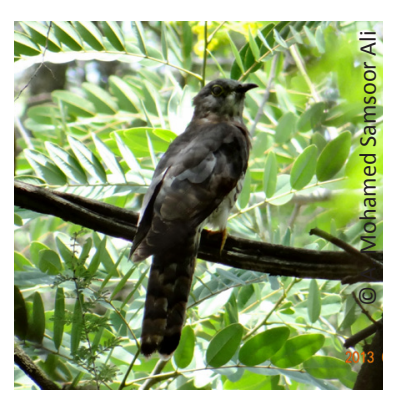

Image 49. Common Cuckoo Cuculus canorus

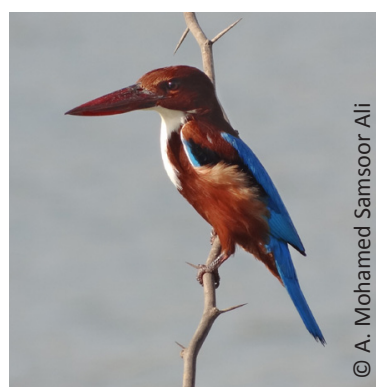

Image 53. White-breasted_Kingfisher Halcyon smyrnensis

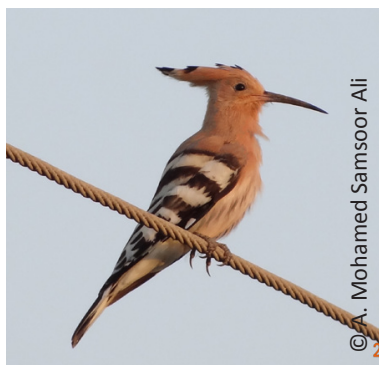

Image 57. Common Hoopoe Upupa epops

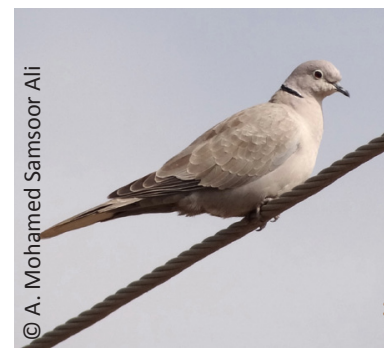

Image 46. Eurasian Collared-Dove Streptopelia decaocto

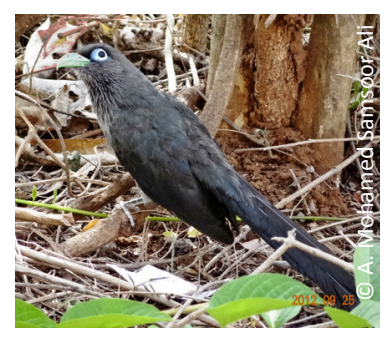

Image 50. Small Green-billed Malkoha Phaenicophaeus viridirostris

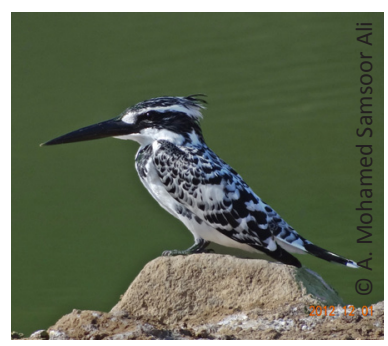

Image 54. Lesser-Pied Kingfisher Ceryle rudis

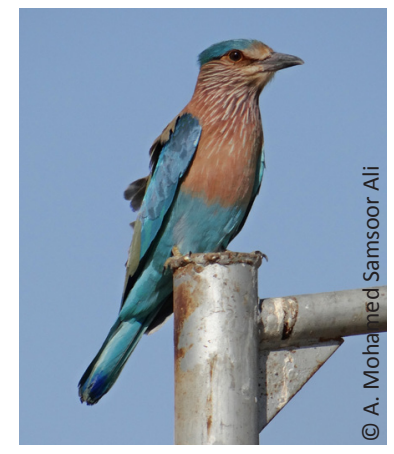

Image 58. Indian Roller Coracias benghalensis 

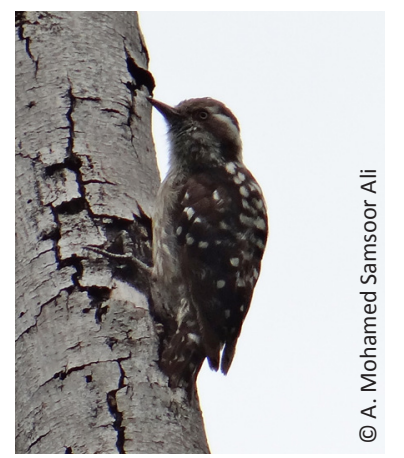

Image 59. Brown-capped Pygmy Woodpecker Dendrocopos nanus

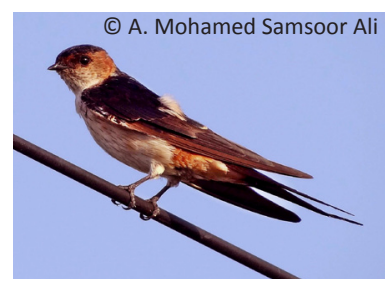

Image 63. Red-rumped Swallow Hirundo daurica

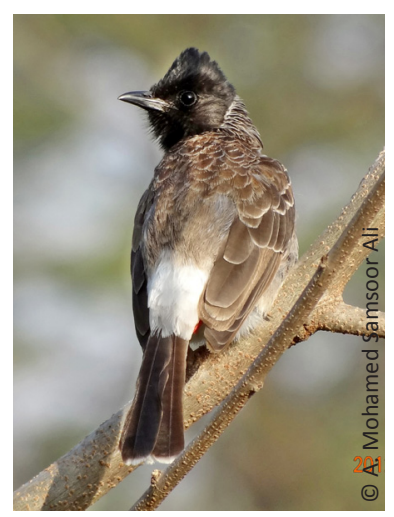

Image 67. Red-vented Bulbul Pycnonotus cafer

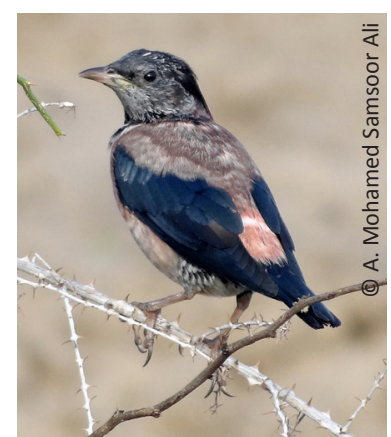

Image 71. Rosy Starling

Sturnus roseus
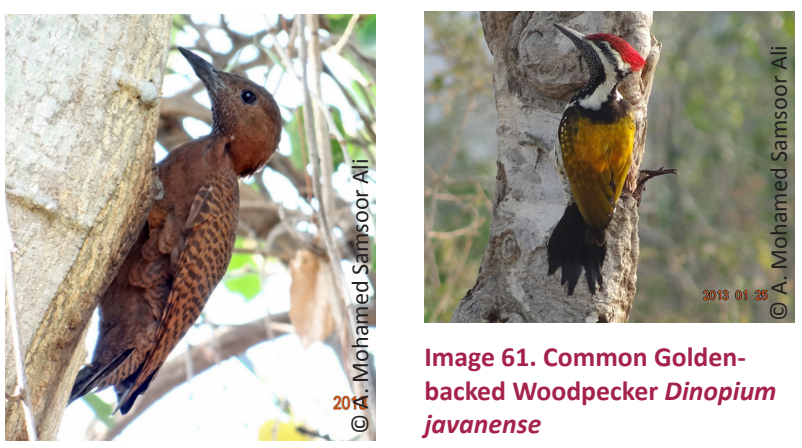

Image 61. Common Goldenbacked Woodpecker Dinopium javanense

Image 60. Rufous Woodpecker Celeus brachyurus

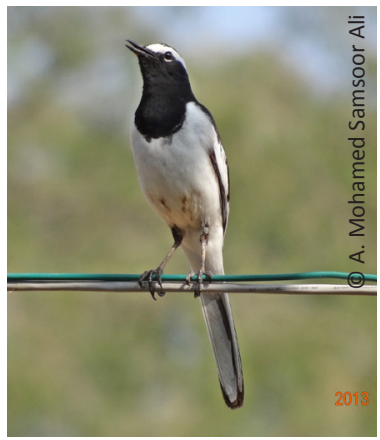

Image 64. Large-pied Wagtail Motacilla maderaspatensis

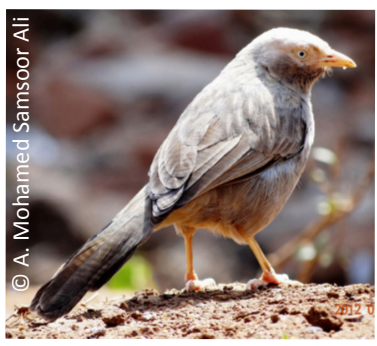

Image 68. White-headed Babble Turdoides affinis

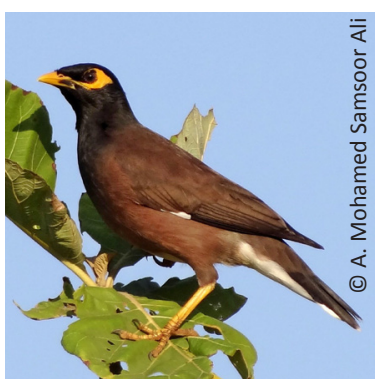

Image 72. Common Myna Acridotheres tristis

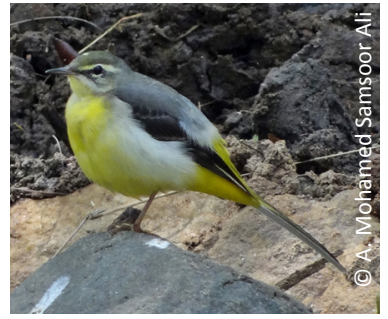

Image 65. Grey Wagtail Motacilla cinerea

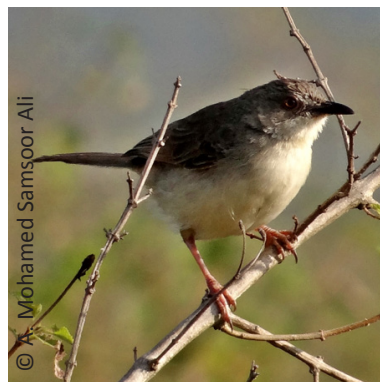

Image 69. Jungle Prinia Prinia sylvatica

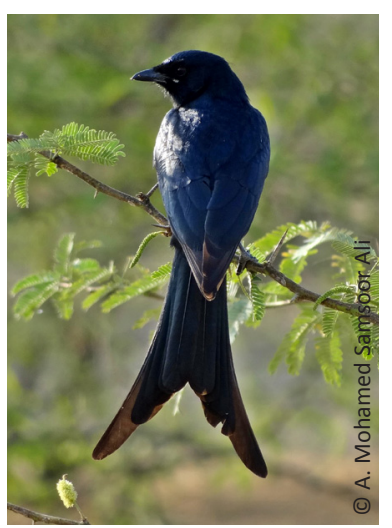

Image 73. Black Drongo Dicrurus macrocercus

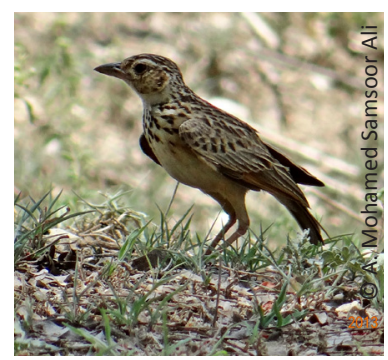

Image 62. Jerdon's Bush-Lark Mirafra affinis

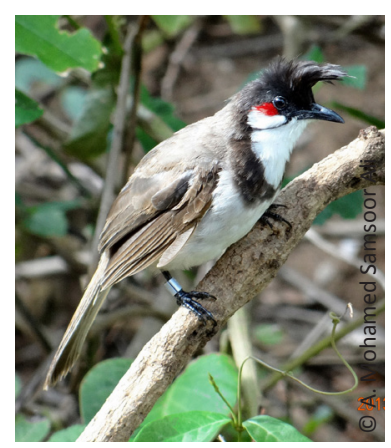

Image 66. Red-whiskered Bulbul Pycnonotus jocosus

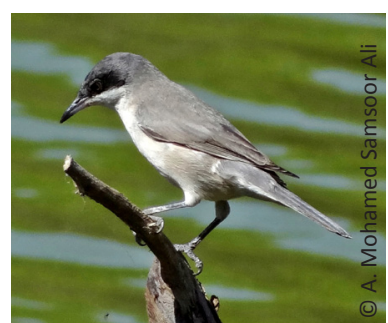

Image 70. Humes Lesser Whitethroat Sylvia althaea

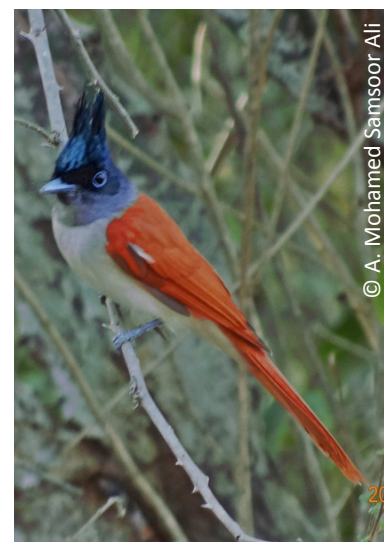

Image 74. Asian Paradise Flycatcher Terpsiphone paradisi 


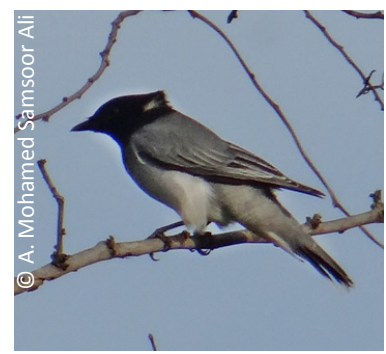

Image 75. Black-headed Cuckoo Shrike Coracina melanoptera

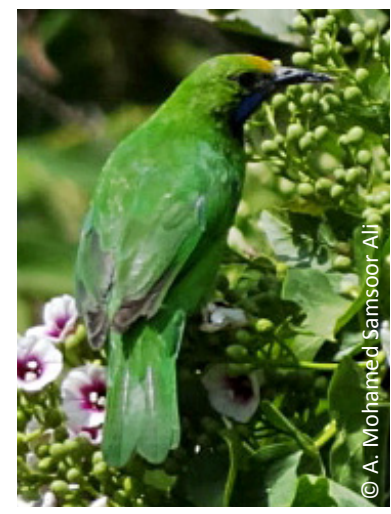

Image 79. Gold-fronted Chloropsis Chloropsis aurifrons

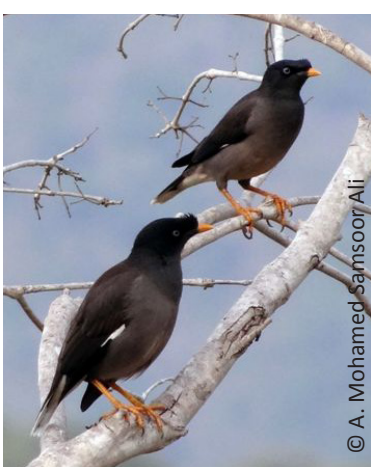

Image 83. Jungle Myna Acridotheres fuscus

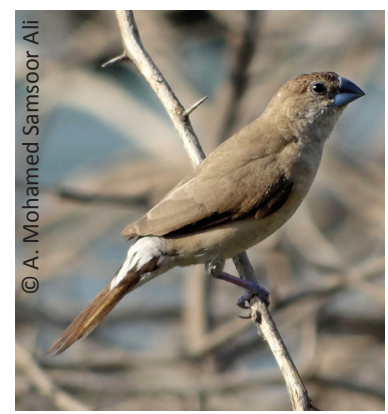

Image 87. White-throated Munia Lonchura malabarica

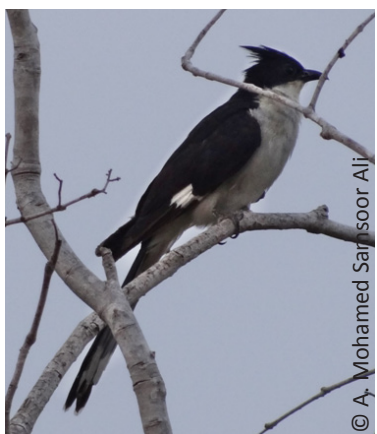

Image 76. Pied Crested Cuckoo

Clamator jacobinus

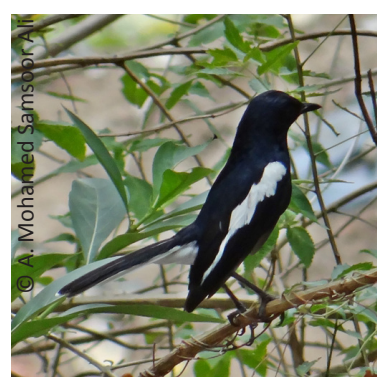

Image 80. Oriental Magpie-

Robin Copsychus saularis

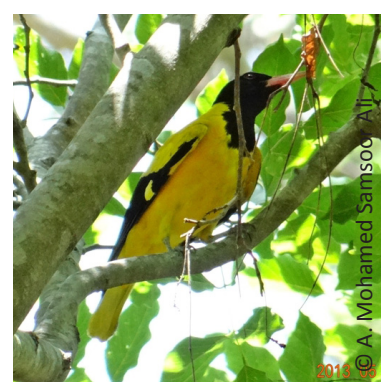

Image 84. Black-headed Oriole Oriolus xanthornus

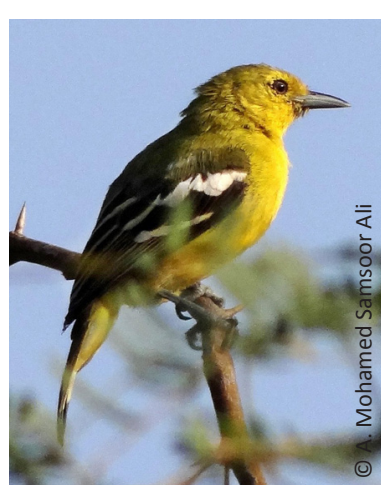

Image 88. Common lora Aegithina tiphia

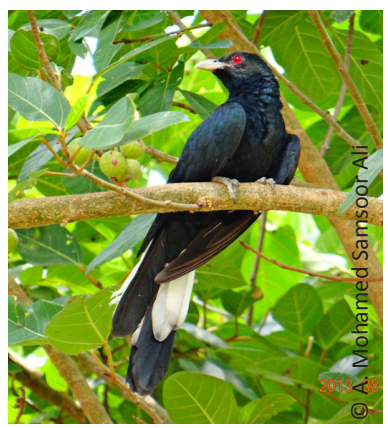

Image 77. Asian Koel

Eudynamys scolopacea

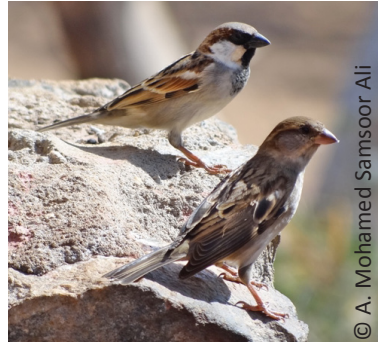

Image 81. House Sparrow

Passer domesticus

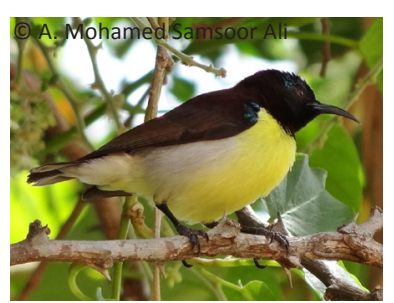

Image 85. Purple-rumped Sun-

bird Nectarinia zeylonica

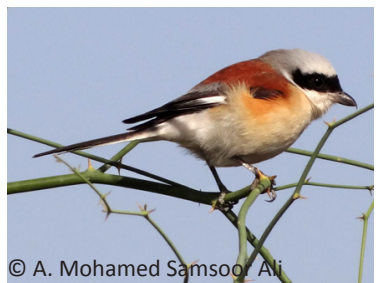

Image 89. Rufous-backed Shrike Lanius schach

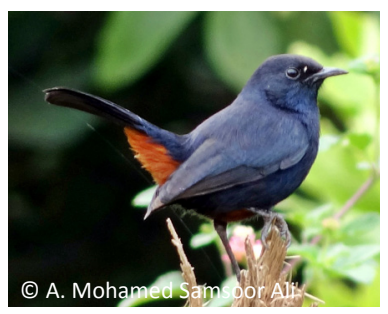

Image 90. Indian Robin Saxicoloides fulicata

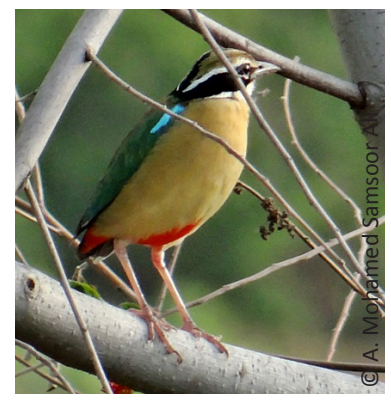

Image 78. Indian Pitta Pitta brachyura

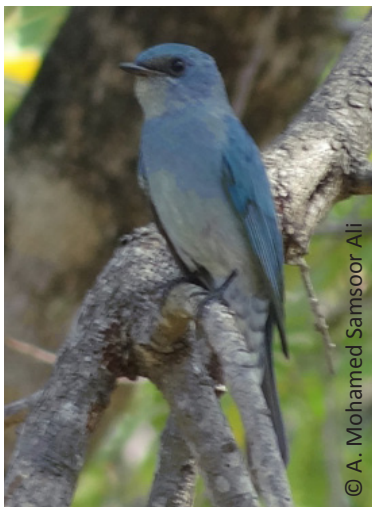

Image 82. Verditer Flycatcher Eumyias thalassina

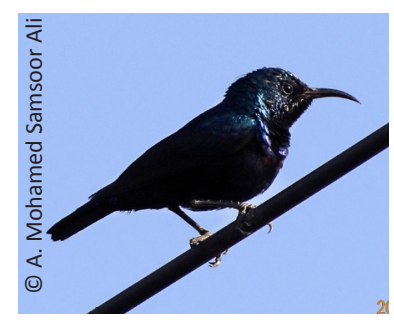

Image 86. Purple Sunbird Nectarinia asiatica

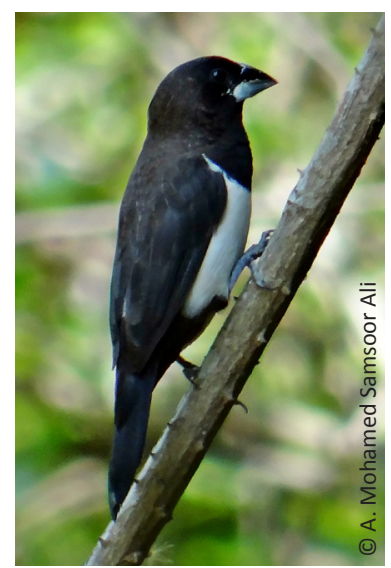

Image 91. White-rumped Munia Lonchura striata 